\title{
Critical analysis of some assumptions and observations on photolytic oxygenesis by plant cells
}

\author{
Kelath Murali Manoj ${ }^{*}$ \\ "Satyamjayatu: The Science \& Ethics Foundation \\ Kulappully, Shoranur-2 (PO), Palakkad District, Kerala State, India-679122. \\ Email: $\underline{\text { satyamjayatu@yahoo.com }}$
}

\begin{abstract}
Kok-Joliot cycle is the acclaimed explanation for photolytic oxygen evolution occurring at the MnComplex of Photosystem II of chloroplasts. Herein, in conjunction with my group's recent critical explorations in this field, an analysis of some pioneers' datasets is made available. The findings pose significant concerns regarding the four-step linear oxygenesis mechanism and pointers are provided for alternative explorative pursuits to unravel the rationale of photolytic oxygenesis.
\end{abstract}

Keywords: Kok-Joliot cycle, Water splitting complex, Oxygen evolving complex, photolysis, oxygenesis, 
A brief introduction to photolytic oxygenesis by green plant cells: Since radioactive tracers incorporated as oxygen atom $\left({ }^{18} \mathrm{O}\right)$ of water gave radioactivity in evolved gaseous oxygen molecules, it was evident that water serves as the source of electrons and oxygen atom. The observation that dark-adapted chloroplasts fail to produce oxygen with a single flash (Allen and Franck, 1955) inspired the direction of research in photosynthesis thereafter. This was also bolstered by the theoretical premise that the oxygen evolution mechanism would be a four-step or four-electron (or four-photon) reaction because:

$$
2 \mathrm{H}_{2} \mathrm{O} \rightarrow \mathrm{O}_{2}+4 \mathrm{H}^{+}+4 \mathrm{e}^{-}
$$

Subsequently, Joliot's, Kok's and select other groups had explored and established new techniques/analyses in the field. When dark-adapted chloroplasts were primed with a "zero" time illumination, the oxygen evolution pattern obtained by the subsequent exposure to a series of light flashes showed a "quartet-periodicity", before attaining a constant steady-state oxygenevolution. This observation was first reported by Pierre Joliot and purportedly explained by Bessel Kok; and it went on to become the paradigm called Kok-Joliot "linear 4-step" model (Joliot et al., 1969, Kok et al., 1970; Forbush et al., 1971; Joliot, 2005; Cheniae, 1993), as captured in Figure 1. In conjunction with the supposition of the " $Z$ scheme of ETC", the mechanism entails that the that the McComplex of Photosystem II (water splitting complex, WSC or oxygen evolution complex, OEC) proceeds through a cyclic change of oxidation states from $S(0)$ to $S(+1), S(+2), S(+3)$; and transiently via $S(+4)$, after/at which it releases oxygen, to regain the "native" $S(0)$. This progression of the MnComplex through the various oxidation states from 0 to +4 completes one cycle in a time frame of approximately $10^{-3}$ seconds. KokJoliot reasoned that the initial periodicity of oxygen evolution was mediated by cooperativity of charges within the OEC (wherein the four $\mathrm{Mn}$ atoms are also aided by the $\mathrm{Ca}-\mathrm{O}-\mathrm{Cl}$ atoms) and labile intermediates within chloroplasts. Essentially, the works implied that oxygen evolution occurs at a unique site alone within the chloroplasts, and that occurred at the designated site after a series of four incremental steps. That is- the mechanism does not permit oxygen release in any state of the complex other than between $S(+4)$ and $S(0)$. This supposition also implies that the experimental findings of DROS within the system are toxic products of unwanted side-reactions. Herein, I shall re-analyze and re-present their own data to investigate if the assumptions and scheme advocated by Joliot/Kok are tenable. 


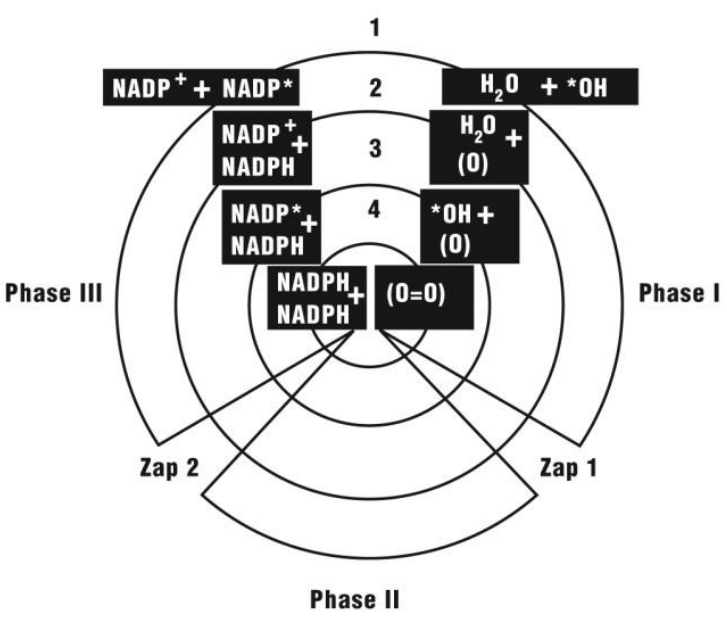

Theoretical ETC

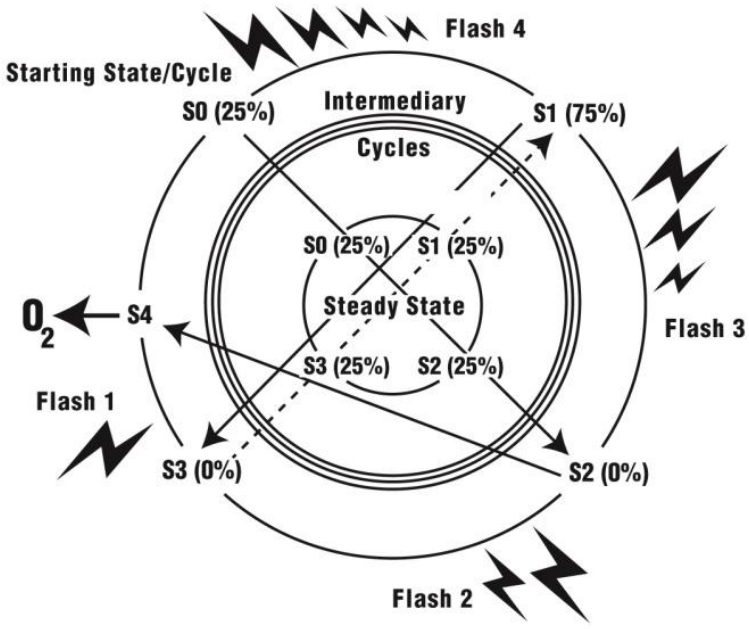

Joliot-KoK Oxygenesis

Figure 1: A schematic representation for the synchronization of four-electron transport and oxygenesis. The progression is clockwise, starting from top-left ( 11 O'clock). In the ETC, through a cascade of steps (shown in the left panel with numbers 1 through 4 , which can also be equated to four consecutive flashes of/within a Kok-Joliot cycle), water loses four electrons, and each electron passes via the three phases and two zaps (one each at PS I and PS II). Protons could be released at any (or specified) moment of the cycle and they are not shown. In the oxygenesis scheme, four flashes are required from S0 state to come to S4 state, leading to the liberation of oxygen. In the starting state, only the S0 and S1 populations of WSCs are believed to be stable, and are supposed to exist at a ratio of 1:3. After $\sim 4$ to 6 cycles, the steady state is attained, where all four WSC populations are at a ratio of 1:1:1:1. Therefore, the starting S0 state can receive 4 flashes (within the first cycle, as shown) whereas the starting S1, S2 and S3 states can only receive 3,2 and 1 flashes respectively. The criss-crossing straight lines show putative double hits, leading to two electron transitions. Triple/quadruple hits are not considered relevant.

Methodology: At the very outset, I would like to affirm that carrying out a critical analysis of the pioneers' meticulous works does not mean I disrespect them in any way whatsoever. I hope that the readers see that I am just trying to search for more meaningful explanations in this endeavor. The arguments presented herein should be seen in conjunction with our earlier critical analyses of the light reaction of photosynthesis; particularly, with respect to the interpretation of Emerson experiment (Manoj et al., 2020). The discussion herein uses the contents of the chapter titled "Oxygen evolution in photosynthesis", authored by Pierre Joliot and Bessel Kok, from the book "Bioenergetics" (Govindjee, 1975) and the particular papers analyzed henceforth within the Results \& Discussion section of this manuscript can be retrieved from the same publication. The respective figures were magnified (keeping the $\mathrm{X}-\mathrm{Y}$ scale ratio intact), printed out, digitalized and re-plotted/re-analyzed with GraphPad Prism. 
Analysis, Results \& Discussion: The essential observations founding this paradigm are (from Antony Crofts' website, UIUC: http://www.life.illinois.edu/crofts/bioph354/lect25.html)-

1. No $\mathrm{O}_{2}$-evolution on the first flash, very little $\left(0.2 X Y_{S S}\right)$ after the second, a maximal yield $\left(2.5 X Y_{S S}\right)$ after the third, and a substantial yield (1.3X $\left.Y_{S S}\right)$ after the fourth (where $Y_{S S}$ is the flash yield in the steady-state). In similar experiments using shorter laser flashes (20 ns) it has been shown that no $\mathrm{O}_{2}$ is evolved on the second flash.

2. A maximum in $\mathrm{O}_{2}$-evolution every fourth flash after the third.

3. A damping of the amplitude of the oscillation.

It can be seen from Figures 2 through 4 that several observations (and inferences thereof) noted by Kok were misplaced. There is no doubt that statistically, the third flash and its periodic repeats afforded high oxygen rates/yields in most cycles of "quartet flashes". (Figures 3 \& 4, panels a through i present the same data sets, the first for 'area under the curve', AUC analysis and the second for evolution in time.) However, in Figure 2, Forbush's data (Kok's own group, on which the model was based!) shows that the peaks were centered on the fourth flash, fourth cycle onwards. In this regard, values within Figure $3 \mathrm{~b} / 4 \mathrm{~b}, 3 \mathrm{~g} / 4 \mathrm{~g}, 3 \mathrm{j}$ etc. are other examples of reaction setups that negate the summated observations. In Figure $3 i$, the $4^{\text {th }}$ flash gave the highest yield within the first cycle itself. The second flash within the first cycle gave significant values in several researchers' experiments (and this is not "very little or negligible" as Kok stated). In Figure 2, one can clearly see that while a few peaks may coincide, the valleys don't. Also, the relative yields of values do not give a correspondence across experiments. For example- in Kok's data within Figure 2 , the $3^{\text {rd }} \& 4^{\text {th }}$ flashes (and their periodic repeats in the next two cycles) gave values higher than steady state rates whereas Joliot's data showed this trend only for the $3^{\text {rd }}$ flash (and its repeats in the next two cycles). Therefore, direct visualizations and deductions from Kok group's data and comparative analyses (with several researchers' data) do not support the prevailing "sequentially ordered and deterministic" paradigm.

Per the Kok model, the ratio of WSC population $(\mathrm{S} 0: \mathrm{S} 1: \mathrm{S} 2: \mathrm{S} 3)$ in the initial state is in the ratio of 1:3:0:0 and the steady-state population is 1:1:1:1 (Figure 1). So, in the first cycle, the fourth flash should have $~ 1 / 3$ yield of the third flash (because first and second flashes gave little oxygen!). This requisite is flouted by practically every researcher's experiment, including Kok's 
own group's data. In Figure 2 above for Kok's group's first cycle data, little oxygen evolution was seen in the first two flashes and the fourth flash gave $\geq 1 / 2$ the oxygen in comparison to the third flash. This must imply that very little double hits occurred in the first two flashes. If they did, we should have seen significant oxygen evolution in $2^{\text {nd }}$ flash itself. Therefore, in the third flash alone, the WSC experienced significant double hits. But then, if it did so, there would have been no way for any loss of oxygen here because a double hit would have taken the ETC through S4, which would have released the oxygen. Therefore, Kok's explanations (invoking a quartet of charged stable/labile states) do not account for the first cycle itself, in Kok's own datasets!
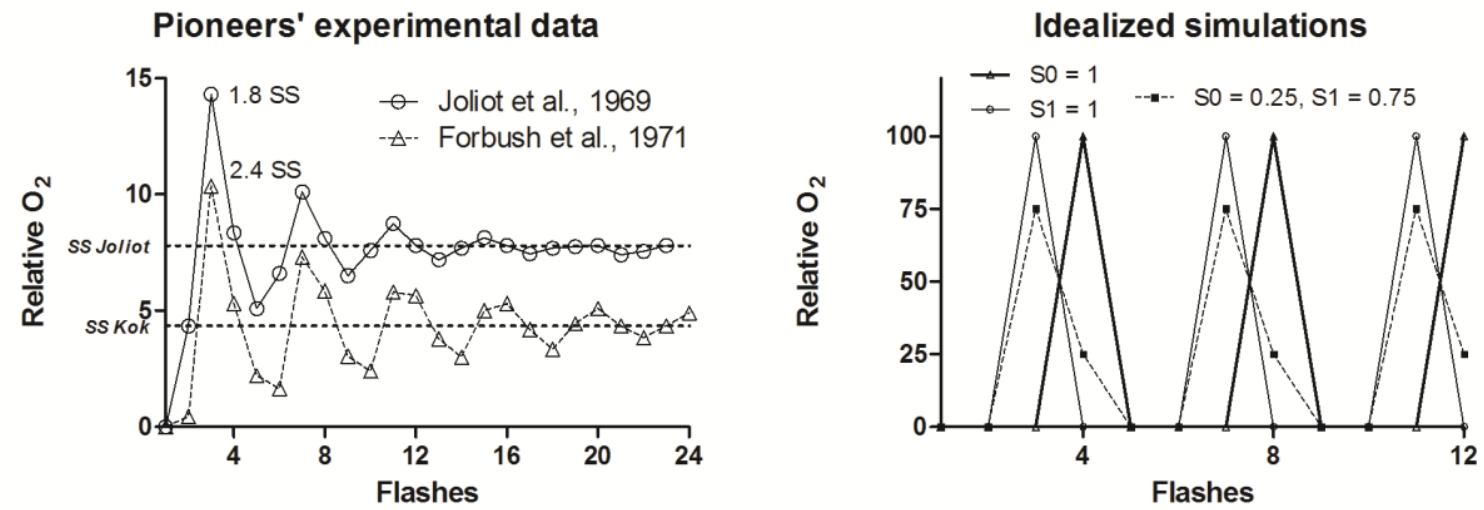

Figure 2: The oxygen evolution pattern reported by Joliot and Kok research groups (when darkacclimatized chloroplasts, pre-primed with suitable light flash) were exposed to periodic light flashes. On the right, simulations are made wherein the initial state is perpetuated as such (sans approach to the "steady state" that is experimentally observed), without double hits and/or misses.

In Kok's data, the second and third cycle show that the $1^{\text {st }}$ flashes' (which corresponds to S3 state) yields are higher than the respective $2^{\text {nd }}$ flashes' (which corresponds to $\mathrm{S} 2$ state) yields. How could misses/double hits explain this outcome (given that going through transient S4 is obligatory!)? It can be seen that in several of these experimental systems, steady state is approached after $4^{\text {th }}$ cycle. Sometimes, even 6 cycles may not be enough to reach the steady state (as seen in Figure 2). Also, if hits and misses were fairly randomized (which is what one would normally expect; given these should be clearly subject to the same parameters through several cycles), there should be little change in the S0 population, the S2 and S3 populations should only increase and S1 population should only decrease. It is very disconcerting to derive the "counter- 
theory" inference that the populations decay/evolve with rather unpredictable cross-overs in several reactions (Figure 4b, 4f, 4g).

In some experiments, the reason why the fourth quartet flashes go higher in oxygen yields (above the third quartet flashes, as the cycles go onwards to steady state) cannot be explained with the Kok model in a forthright way. (For doing so, one has to assume that the hits, misses and double hits have some rather skewed values for each one of these transition steps/experiments. That is what Kok's model solicits by assuming different $\alpha$ (index of misses) and $\beta$ (index of double hits) values for fitting each experimental data. Also, the initial stable values of the four states must be different in each setup [and not just $\mathrm{S} 1(3): \mathrm{SO}(1)$, as Kok assumed for a particular setup]. This practice would be similar to building and fitting a new theory to the experimental observations every time. We need to have a relatively simple theory that can explain a multitude of observations, at least on a qualitative scale. The much-acclaimed Kok's data fitting in Figure 2 of Forbush et al. (1971) has this problem evident (only the experimental values are shown in Figures $2,3 \mathrm{f}$ and $4 \mathrm{f}$ herein). While the experimental data shows peaks only at the $3^{\text {rd }}$ quartet flashes within the first three cycles, the fit model shows peaks at $3^{\text {rd }}, 3^{\text {rd }}$ and $4^{\text {th }}$ quartet flashes of cycles 1, 2 and 3 respectively. Clearly, the observations display qualitative disagreement with the theoretical foundations. As seen, the ratio of steady state oxygen yield to the first cycle's $3^{\text {rd }}$ and $4^{\text {th }}$ flashes' yields, the number of cycles required for arriving at steady state, correspondence of relative rates within and across experiments, etc. varied significantly across groups and within groups. Given the deterministic theoretical basis of Kok's model (and ETC scheme), one would imagine that the above mentioned indices should have some common patterns and at least some experimentally observable "constant" signals should be derived. Clearly, the diverse observations do not conform to "expected reproducibility norms" that the currently prevailing deterministic ETC and oxygenesis scheme would dictate.

The only consistent observation that can be tangibly derived across different groups is- AUC for each cycle is practically constant (save the setups incorporating additives). This must mean that "misses" and "double hits" schemes are "quantitatively insignificant" in the overall scheme. While RC's 1-electron excitability is in small (sub-nano second) time-scales, its ability to get "double hit" depends on the "transferability" aspect, which is dependent on the slow 2-electron 
cycle of the bound/mobile quinones! [Also, double hits of S3 $\rightarrow$ S1 are antithetic, not compatible with the ETC model. This is because it is not WSC that is directly under photo-electronic effects. It is the PSII's RC that is subjected to photonic excitations. In turn, the ETC that the RC is subjected to is inherently a one-electron transport system (without any transient pools to donate to or buffer with!) with two-electron transfers as rate-bottlenecks. An electron from WSC can move onward only if the ETC completes a full cycle. That is, a WSC in S3 state would have to go through S4 state before coming to S1 state. If it gets to S4 state, the connectivity breaks because oxygen is released and two more water molecules have to bond. Since the WSC is not subject to the photoelectric effect directly, double hits of this kind are merely "mathematical exercises" with little theoretical or experimental significance.]

Very importantly, per the prevailing ETC/oxygenesis model, there is no scope for charge conservation in the system, because the relay agents are ordered and sequential (in series), with either single or double charge bearing capacities (excluding the WSC). (Also, the motility of quinones are rate-limiting!) The current mechanistic purview does not permit charge to be retained or passed on from one cycle to another because each component is of a defined capacity and role. Saying that an initial photo-stimulation exercise enables the buildup of quinone pool is antithetical because there is nothing that stops the quinones from going through with the full cycle thereafter (which is supposed to take sub-millisecond time frames, compared to the seconds and minutes of the latency that ensues before the flashes are given!). Further, when the Kok-Joliot explanations were put up, the structures of the photosynthetic complexes were not known. The ability of the WSC/OEC to go through periodic cycles of electron transfer on the "hit/miss" of photons hitting RC of PS II is an untenable/unsubstantiated proposal. That is, the ETC connectivity through the Z scheme cannot be coordinated with the Kok-Joliot cycle, in any foreseeable way. This is because there is little physical connectivity for the hit/miss phenomenon to lead to a periodicity in the oxygen evolution profiles as seen in the Joliot cycles. Furthermore, this aspect is elaborated in the discussion below. 

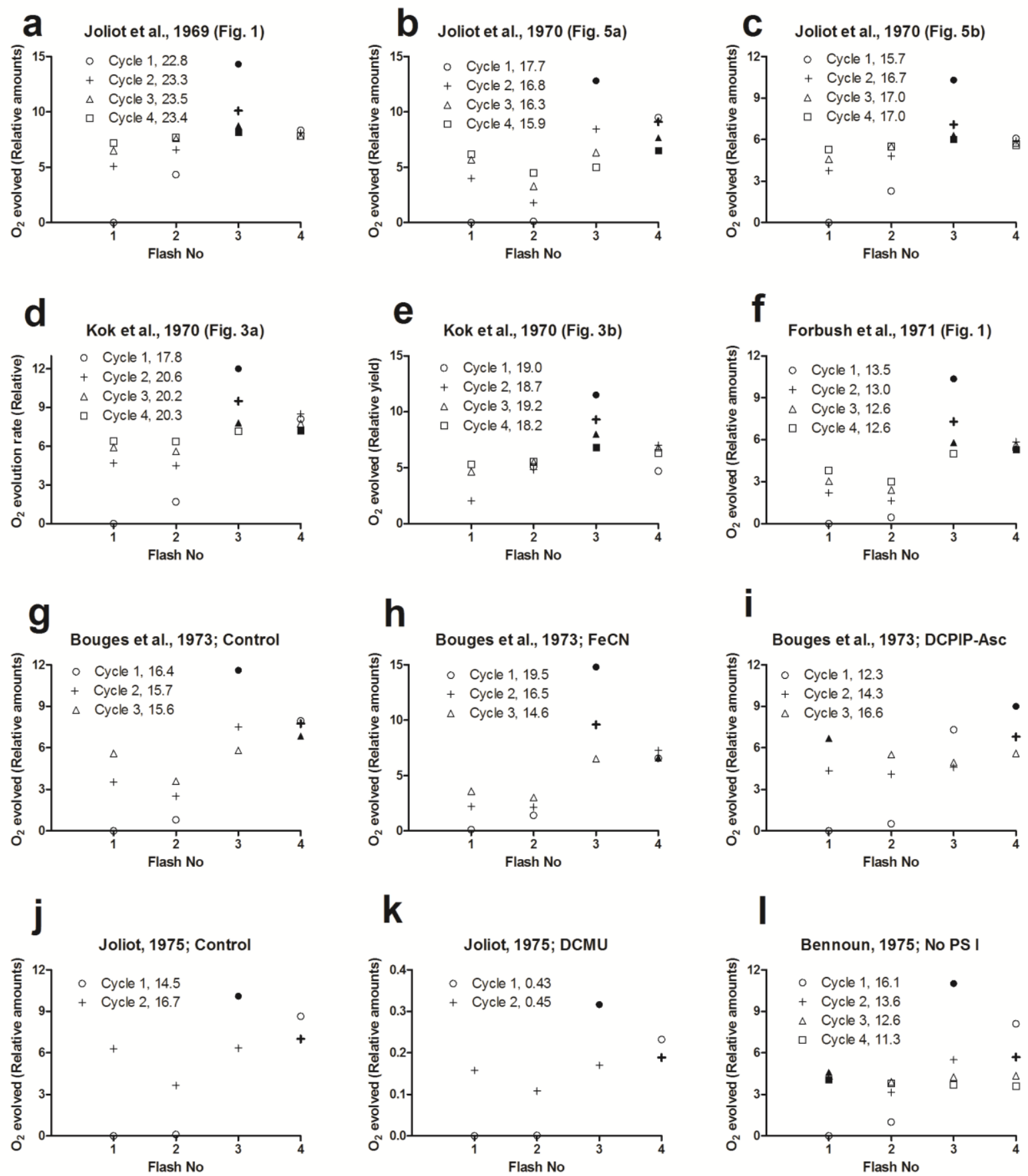

Figure 3: Cycle-wise analyses of oxygen evolution from pioneering researchers' data. In spite of the observations that the absolute value of amplitude changes, and also the peak within a quartet could lie on $3^{\text {rd }}$ or $4^{\text {th }}$ flash, the area under the curve (AUC) remains constant through cycles (for experiments that did not incorporate additives), throughout the series of quartet flashes. AUC values are given for each cycle and the peak in each cycle is highlighted with a filled and emboldened symbol. 

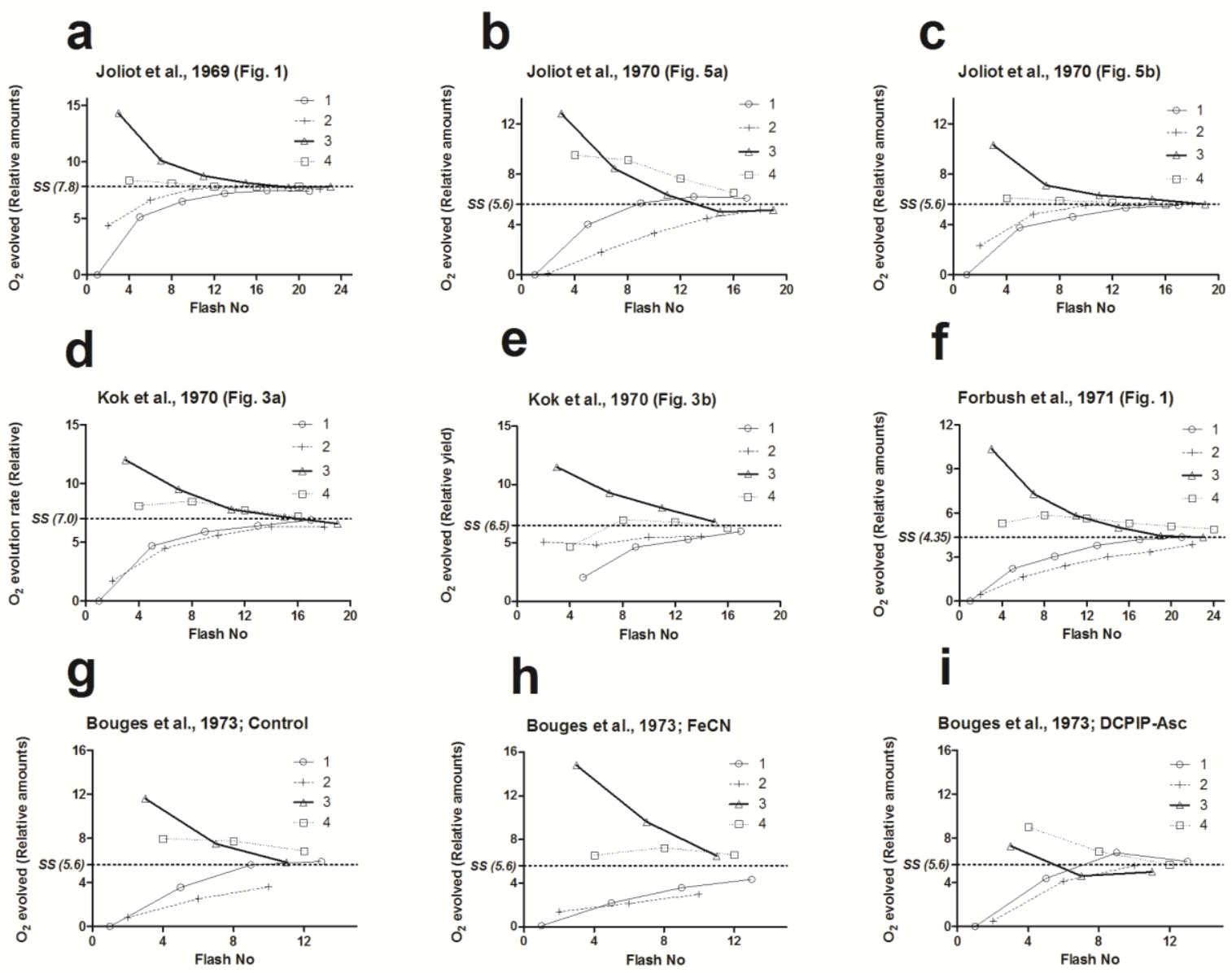

Figure 4: Analyses of pioneers' data for temporal-phased oxygen evolution with respect to multiples of flash numbers 1 through 4. (The values of y axes in the panels of the first two rows are valid for comparison within the given panel only.)

The dark adapted chloroplasts were primed with a suitable amount of light (a single flash followed by some "resting time"), before they were subjected to the series of flashes (numbered 1 onwards, as shown in Figure 2) that were taken as "consequential". If a good starting point or consistency was desired with respect to experimental requisites, the only way it could have resulted is to have dark acclimatized chloroplasts to start off with, and having no "priming light" provision. If the explanation was based on a postulate that only one or two of the four WSC intermediates were initially stable, then what was the purpose of doing the pre-priming exercise, which would mess with that proposal of stability? Would not this "light priming" mess with the interpretations of periodicity and stability? This query is of paramount importance. The explanation afforded by Kok is that this single priming step affords the build-up of charged 
intermediates, so that a sequential relay can be set in thereafter. How can this be compatible with the theoretical assumptions of the ETC-oxygenesis cycles, per se? The assumption of prevailing ETC is founded on a linear charge transfer scheme with definite and sequential roles to each participant. The oxygenesis is supposed to be based on only four charge-able/discharge-able states of the system (and that being the WSC!). There are no generic roles or "buffering or reservoir" agents within. While the "intervening dark period" between flashes were in $\sim$ second scales, several intermediates' stability time-scales would be envisaged to be in sub-microsecond (or surely, in sub-millisecond scales!) time scales. Then, how is the assumption of "4 distinct electronic states of WSC and their stabilities" used to explain the observation of arriving at a steady state from an initial periodicity? Most importantly, when the overall observation is supposed to be interpreted on a "one-flash, one electron" scheme, the explanation is based on "one-flash, zero/one/two electron" scheme (as the model assumes misses, single and double hits)! So, not only were the observations inconsistent with the explanations, the assumptions and explanations thereof were paradoxical and antithetical too! Thus, when scrutinized up close, it is seen that Joliot-Kok model is something quite like the chemiosmosis model- a mirage, neither making any holistic sense nor affording any purpose (other than fitting a curve to some equation).

Fig. 2 of Kok et al, 1970 is crucial to arrive at the crux of the issue here. It shows the oxygen evolution profiles by dark-acclimatized chloroplasts under a steady monochromatic light source, starting with nil or a single priming flash. The unprimed sample traced a sigmoid curve initially, significantly lagging behind the somewhat hyperbolic trace of the primed sample, although both traces achieved almost identical steady state values (>60 seconds). (Though the initial rate of the unprimed sample lagged behind the primed one, the former's trace caught up and exceeded the corresponding value of the primed sample, after crossing over at $\sim 5$ seconds.) The primed and non-primed samples reached their respective maximal values at $\leq 2$ and $\leq 8$ seconds. Within the first 10 seconds, both non-primed and primed samples showed higher oxygen evolution ( 7\% and $12 \%$ respectively) with respect to the steady state values. The protracted phase of the constant "steady state" value of oxygen evolution in the later times argues against inhibitions or deactivations. Therefore, it is clear that the correlation of oxygen detection to the "priming step", "maximal rate" and "steady state" is more complex than what is apparent. I have seen such time- 
course profiles in simple heme-enzyme catalyzed peroxidation reactions. These profiles surely indicate a dynamic and temporally evolving interaction scheme of one-electron intermediates within milieu.

In Figure 1 of the very same paper by Kok et al (1970), the scenario analogous to the one shown in Figure 2 is extended to a series of priming flashes, from 0 to 7 (which have been duly labeled therein). It is seen that in almost all the samples, the steady state is achieved after $\sim 6$ seconds (save the unprimed sample, which achieves steady state after $\sim 8$ seconds). The point to note here is that samples that received 3 and 7 preceding flashes start off with higher oxygen evolution, which dips to lowered rates than the steady state values during the time period of $\sim 1$ to 5 seconds of illumination. Samples with 2 and 6 priming flashes show much higher values initially (than steady state yields), which falls rapidly as the illumination time increases, finally stabilizing at steady state values (never going lower than the steady state values). These data could now be compared to Figure 4, where the oxygen evolution profiles of diverse workers have been presented as a function of the flash number (post the unique priming flash). The equivalent profiles are traces 1 (and 5), 2 (and 6), 3 (and 7), and 4 of Fig. 1 of Kok et al. (1970) and the connected profiles of traces numbered 1,2,3, and 4 of each one of the nine panels of the Figure 3 of this manuscript. Even if we concede to Kok's inadmissible assumptions, the lack of one to one correspondence of data flouts the "stability of S0-S1-S2-S3 states of WSC" explanation.

An honest admission here, for the ensuing discussion: I am not confident about my concerns on protocols here because of a lack of "clear understanding" of the experimental procedures adopted. (The early papers by Joliot were in French and also, the experimental setups are unique to the field.) However, here are some samples of my doubts.

The rate calculations appear to be misplaced. How can initial rate be calculated with any experimental accuracy when the profiles are starting at different initial points? In the Figure 10 of Forbush et al, the data cannot be used to calculate rates. While curve 1 starts at a $\mathrm{Y}$ value of 0.2 , the curve 2 starts at 2.2. The value of $\mathrm{Y}$ axis at the initial point of experimentation cannot be taken for rate calculation. 
Joliot claims a response time of $10 \mathrm{~ms}$ for the electrode (Joliot \& Joliot, $1968 \mathrm{BBA}$ ) for the oxygen assay profile in Figures 7 and 8 of their paper. In Figure 7, Curve 1 is the unprimed sample, which is compared to Curves 2 and 3 in which light-priming was done with different setups. In all three experiments, the curves attain the amplitude of $\sim 5$ (Curve 1 takes $\sim 50 \mathrm{~ms}$ whereas curves 2 and 3 reach the amplitude in $\sim 15 \mathrm{~ms}$ and $\leq 10 \mathrm{~ms}$ respectively) and subsequently, all curves show identical decay profiles to reach the minimal value of $\sim 1$ after 1.8 seconds. Therefore, except for the time required to achieve the amplitude value, the time course of oxygen detection showed almost identical profiles for the two flashes, and only the "initial time" for attainment of the amplitude signal of the electrode changed across samples. I find that in the context, we needed to have an accurate measure of the yield of oxygen per flash, and not the initial rate per se. Else, we should have observed different amplitude values and decay curves for the different samples. Therefore, if the area under the electrode response decay curve is taken as the yield, it would give only a small variation of $<10 \%$ signal/output between the three experiments. At low temperature $\left(1^{\circ} \mathrm{C}\right),<400 \mathrm{~ms}$ was required for attaining the saturation value of oxygen released when subjecting chlorella cells to bright flashes of light [Emerson and Arnold (1932)] and the reaction half life was $<40 \mathrm{~ms}$. At about $25{ }^{\circ} \mathrm{C}$, this half-life value can be approximated to be $\sim 4 \mathrm{~ms}$. Therefore, the overall reaction of oxygen evolution falls within the rough experimental range of the probe employed (whose response time was 10 millisecond). This would not be the best way to do an experiment! [And this would in turn mean that regardless of which flash number we consider, similar oxygen yield is obtained! Therefore, the multiplicity of "four illuminations leading to peak oxygen evolution" that was taken to be a yardstick for oxygen evolution, would be a sheer fallacy. Also, the first priming illumination(s) were taken out of the picture to derive an experimentally and theoretically aesthetic value (of quartets). Since oxygen evolution via the deterministic scheme would be from 2 molecules of water, a primed thought process had dictated the periodicity of oxygen evolution, and this expectation was fulfilled in the pioneers' experiments/inferences.]

When we are talking about dark incubation times in $10^{3}$ seconds and flashes' separation times in seconds and electrodes response time in $10^{1}$ milliseconds and stabilities of the intermediates are the main consideration (which is currently believed to be less than millisecond ranges), my 
argument does not seem irrational. If the elementary 4-state model of Kok was true, we should have observations of prolonged oscillations, as defined by the idealized profiles in Figure 2.

In the prevailing ETC scheme, there is no provision for conservation of electric charges from an initial state (whether dark acclimatized or primed with a definite amount of light) or across "quartet flash" cycles. There are no "electron traps" that are called upon to justify the priming flash. Therefore, the "conservation of charge" observation or explanation cannot be assumed or called upon for the prevailing ETC scheme. (One cannot invoke an antithetical assumption in the middle of a derivation!)

Joliot et al (1968) had computed a time period of $0.8 \mathrm{~ms}$ between the photostimulation event and release of oxygen. (Let us recapitulate that his process would necessitate a 4-electron relay through the ETC.) Kok et al (1969) and Stiehl and Witt (1969) reported a half-time of $~ 10$ ms for photoreduction of quinone pool. How could a reaction cycle with a slow bottleneck lead to a much faster reaction? Clearly, the purported elaborate Z-scheme ETC cannot be the operational mechanism!

Since the ETC cycles around in a time-frame of a few milliseconds, it can only process about a few hundred photons in a second. The same value would also be taken as the functional processing time within the RC. (Even if some excitation and charge transfers occur in nano-pico second time-scales, the electron transfers at several steps are in millisecond ranges. Since the system has finite resources, the steady state scenario would come to the afore-mentioned status. This would lead to the following statistical scenario: one chlorophyll molecule may only absorb a photon per second of the few hundred micromoles of photons that it would get.) Therefore, the prevailing assumptions cannot explain the observations. The conservation of charge can only be explained by a highly randomized/stochastic mechanistic model for oxygenesis, which does not have the sequential limitations of the current understanding.

Bouges et al (1973) data (Figure 4g-i) shows the interesting observation that $\mathrm{FeCN}$ (ferricyanide, an oxidant) gave higher oxygen yields in the initial phases which went down as the cycles progressed, whereas the anti-oxidant ascorbate gave low AUC in the initial phase, which 
increased as cycles progressed. In the presence of ascorbate, the oxygen yield variation amongst flash numbers was lowered and the reaction approached steady state quicker, when compared to the control. The effect was quite the opposite in the presence of FeCN. This counters the specific electron transfer and relay concept of Z-scheme.

As per the Kok model, the state transitions theoretically occur within $10^{1}$ to $10^{3} \mu$ s and the overall cycle itself gets completed within the same order of time as a/the limiting step $\left(10^{3} \mu \mathrm{s}\right)$. Experimentally, the flashes' durations are in the ranges of $10^{6} \mu \mathrm{s}$. Therefore, one could consider that there is ample scope for multiple hits and misses. However, in robust experimental systems and those lacking additives (Figures $3 \& 4$, a through $\mathrm{g}$, and $\mathrm{j}$ ), the area under the curve (AUC) analysis for each cycle remained constant $(\sim \pm 15 \%$ variation, within a given experiment). This statement negates the assumptions/interpretations for the introduction of constants like $\alpha$ and $\beta$ (accounting for the so-called misses and double hits) for explaining the periodic waning and "lowering amplitude". That is- there were no losses within the system as it approached the steady state (and "damping of amplitudes" would only be expected in a system with several states and finite probabilities for crossovers). If there is any kind of miss (with respect to any significant/expected event), we cannot note a conservation of charge through the cycles. The system is in dynamic equilibrium from the starting state until it reaches the steady state (and beyond). Further, though multiple hits seem a theoretical possibility with respect to the excitability of a photosystem, relay of an electron from water to the reaction center must occur within microsecond time scales.

Summating: It is imperative that one understands that a given set of experimental observations could be fit into a wide variety of mathematical equations/theories (with appreciable $\mathrm{R}^{2}$ values) and such a fitting of experimental data is not a projection/prediction of accuracy of the hypothesis/data. For example (as shown in Figure 5), when a simple non-linear regression analysis of concentration versus activity is taken, one can simulate a Michaelis-Menten equation for experimental data (of 10 points) varying through typically one decade of concentration. Now, this is the equation of a typical hyperbola, which can be easily fit into a parabolic (shown here) or elliptical/catenary curves (not shown). Unlike the assumptions involved in M-M kinetics, totally irrelevant exponential associations with one or two binding site considerations could also 
give excellent fits to the data points. So, how does one know which of these fits could be correct? Only by a meticulous and critical analyses of both the theoretical foundations and experimental data, we can approach a more "truthful" and reasonable explanation.
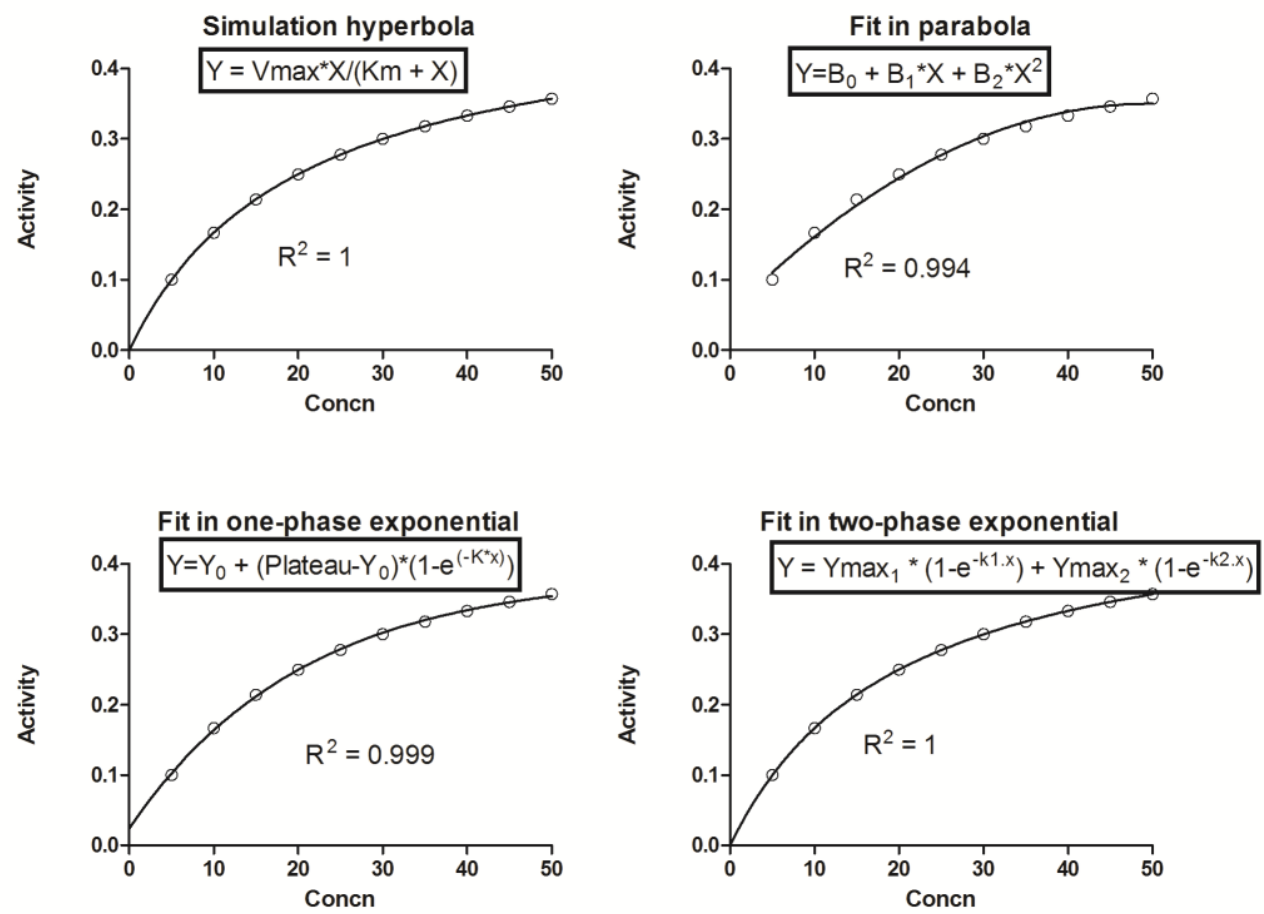

Figure 6: The points correlating and connecting a theory with experiment, a graphical argument

In another perspective, let's say that two spheres alpha and beta attract each other. Now, the attraction force can be based on mass or opposite charges (or it could be due to both or also due to some other reason). Both forces of attractions could be represented by the generic equation:

$\mathrm{F}=$ Constant. (alpha) $.($ beta $) /(\text { distance })^{2}$

In the equation above, alpha and beta could stand for either mass or charge attributes. Now, if we don't have a good idea of the mass or charge or distance parameters involved, we really cannot say (based purely on the numerical values of the constant) as to which force, gravitational or electrostatic (or both!), governs the attraction. Therefore, the fact that an equation could theoretically describe a trace well does not really mean much if the mechanic assumptions and the factual values of the variables don't fit the actual structure-function premises of the system. 
Therefore, what Kok achieved is the fitting an experimental data curve to a quantitative expression/model based on certain mechanistic assumptions that may have little grounding in reality. A Markov chain-based solution for the graphical problem (Shinkarev, 2003) has a stochastic/probabilistic approach which could explain the rather "chaotic" results. However, for having a solid theoretical relevance, we must resort to a different set of starting assumptions and systemic parameters/variables. In which case, it would then be mandated that the outcome therein is interpreted in a different way too.

Figure 6 below shows simple evidence to the statements above. In a reaction system with faulty

or dysfunctional PS I, there was no steady-state achievement, but a gradual decrease in oxygen evolution rate over cycles. Clearly, per the prevailing concepts, oxygen evolution is a function of PS II alone and we see that the periodicity is no more, even at the second cycle (even if we overlook the production of oxygen in the $2^{\text {nd }}$ flash of the first cycle)! Such observations dictate the application of different theoretical premises.

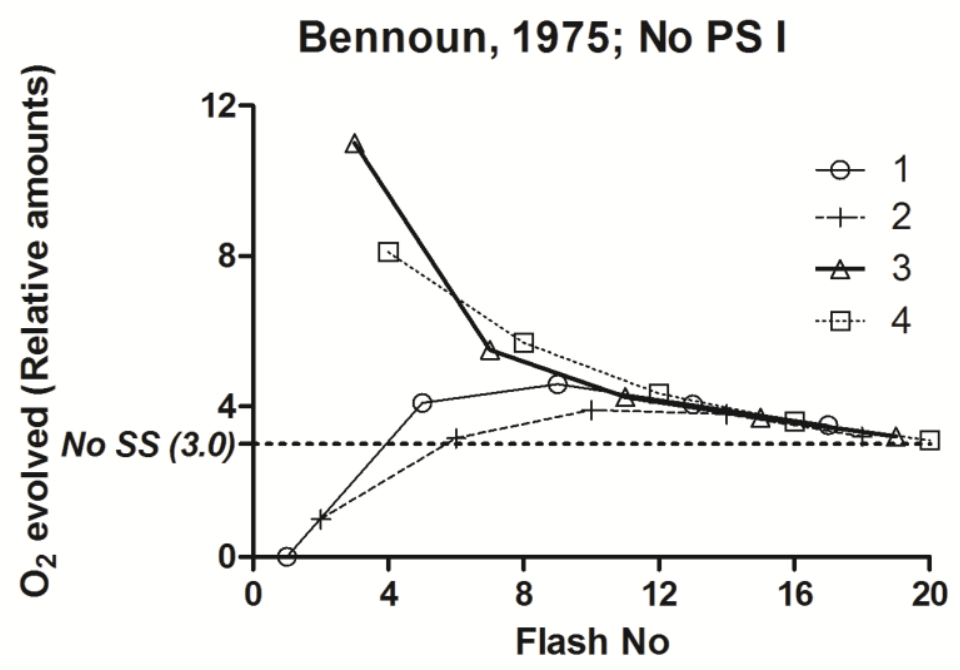

Figure 6: Oxygen evolution in a modified Z-scheme setup

Conclusions: The concerns regarding the Kok-Joliot experimental scheme can be listed into the following points-

1. Some key observations were not factually consistent across the work-groups (and sometimes, within the same group too) and some observations were overlooked. 
2. Some mathematical/chemical assumptions used for the modeling have no theoretical foundations in structure-function correlation.

3. The experiments were conducted and interpreted with a presumptive and misplaced theoretical basis/bias.

4. Some of the adopted protocols could be questioned, as the analytical tools used fell short of experimental requisites.

If we imagine the experimental setup to be an oxygen-limited environment (at the initial state) and also consider that oxygen generation facilitates further catalysis within the system, the initial periodical wavering and the later steady-state values for oxygen can be explained with alternate/alternative perspectives. If a single MnComplex bound two molecules of water to release a single molecule of oxygen at a unique step, we cannot explain why- high partial pressure of oxygen does not lower the rate of oxygen evolution (Haumann et al., 2008) and photosynthesis is lowered at high light intensity and oxygen concentration (Warburg effect). Also, if we imagine that a photo-activated electron-deficient species abstracted an electron from hydroxide ion, it would give hydroxyl radical (Manoj et al., 2020), which could react with other DROS (generated with the priming flash!) to liberate oxygen (Manoj \& Bazhin, 2019) without going through a four-step reaction (e.g. via ${ }^{*} \mathrm{OH}+{ }^{*} \mathrm{OOH} \rightarrow \mathrm{H}_{2} \mathrm{O}+\mathrm{O}_{2}$ ), thereby explaining oxygen evolution in $2^{\text {nd }}$ or $3^{\text {rd }}$ flashes. Such mechanisms (Manoj et al., 2020) are more probable and worthy of exploration.

Declarations: The work was powered by Satyamjayatu: The Science \& Ethics Foundation. KMM thanks Vivian David Jacob for the artwork of Figure 1. A major part of this write-up was presented as Supplementary Information for my elaborate critical exploration on the photosynthetic mechanism, disseminated as a preprint with the following details- Manoj, K. M. (2019, February 5). Oxygenic photosynthesis: Critiquing the standing explanations and proposing explorative solutions based in murburn concept. OSF Preprints. https://doi.org/10.31219/osf.io/3mzfp. Since 150 downloads down the lane did not lead to any reorientation in the field, I have little choice now and have to bring out the information to a greater attention of research community. This is done with the hope that it would aid more inquisitive pursuits. 


\section{References:}

Allen FL, Franck J. (1955) Photosynthetic evolution of oxygen by flashes of light. Archiv. Biophys. Biochem. 58, 124-143.

Cheniae GM. (1993) A recollection of the development of the Kok-Joliot model for photosynthetic oxygen evolution. Photosyn. Res. 38:225-227.

Forbush B, Kok B, McGloin M. (1971) Cooperation of charges in photosynthetic $\mathrm{O}_{2}$ evolution1. Damping of flash yield oscillation, deactivation. Photochem. Photobiol. 14:307-321.

Govindjee. (1975) Bioenergetics of photosynthesis. Academic Press, New York.

Haumann M, Grundmeier A, Zaharieva I, Dau H. (2008) Photosynthetic water oxidation at elevated dioxygen partial pressure monitored by time-resolved X-ray absorption measurements. Proc. Natl. Acad. Sci. USA. 105:17384-17389.

Joliot P, Barbieri G, Chabaud R. Un nouveau modele des centres photochimiques du systeme II. (1969) Photochem Photobiol. 10:309-329.

Joliot P. Period-four oscillations of the flash-induced oxygen formation in photosynthesis. (2005) In Discoveries in Photosynthesis. Eds. Govindjee, Beatty JT, Allen JF. pp 371-378.

Kok B. Forbush B, McGloin M. (1970) Cooperation of charges in photosynthetic $\mathrm{O}_{2}$ evolution1. A linear four step mechanism. Photochem. Photobiol. 11:457-475.

Manoj KM, Bazhin N. (2019). Murburn precepts of aerobic respiration. OSF Preprints. https://doi.org/10.31219/osf.io/hx4p9

Manoj KM, Bazhin N, Parashar A, Gideon, D A, Jacob V D, Haarith D, Manekkathodi, A. (2020) Murburn precepts for the light reaction of oxygenic photosynthesis. OSF Preprints. https://doi.org/10.31219/osf.io/95brg

Shinkarev VP. (2003) Oxygen evolution in photosynthesis: Simple analytical solution for the Kok model. Biophys. J. 85:435-441. 